\title{
Mean light curves of long-period variables and discrimination between carbon- and oxygen-rich stars
}

\author{
M.O. Mennessier ${ }^{1}$, H. Boughaleb ${ }^{1,3}$, and J.A. Mattei ${ }^{2}$ \\ 1 Université Montpellier II, Groupe de Recherche en Astronomie et Astrophysique du Languedoc, Unité associée au CNRS, \\ F-34095 Montpellier Cedex 5, France \\ 2 American Association of Variable Star Observers, 25 Birch Street, Cambridge, Massachusetts 02138-1205, U.S.A. \\ 3 Université des Sciences El Jadida, Maroc
}

Received September 13; accepted November 16, 1996

\begin{abstract}
Using 75 years of AAVSO data, mean light curve parameters of a sample of 355 long period M, S, and $\mathrm{C}$ mira and semi-regular variable stars are investigated. We present a classification of the light curves of LPVs into 6 distinct groups. Combining this classification with IRAS colors makes it possible to distinguish oxygen-rich from carbon-rich miras. ${ }^{1}$
\end{abstract}

Key words: stars: AGB, post-AGB - methods: data analysis — stars: carbon — stars: variables

\section{Introduction}

The long-period variables ${ }^{2}$ (LPVs) located on the Asymptotic Giant Branch (AGB) represent an important stage of stellar evolution in which some are progenitors of planetary nebula while others are directly evolving to the white dwarf stage (see the reviews by Habing 1990, 1996, and Wood 1990). They play a major part in galactic evolution through enrichment of the interstellar medium in heavy elements via mass loss.

Several investigators (e.g. Whitelock et al. 1991) have studied correlations between observed quantities such as the period and amplitude of light variation, asymmetry of the light-curve shape (rise time expressed as a fraction of the period), the IR excess of the star and its $J H K L$ colours, or parameters derived from the IRAS (Infrared Astronomical Satellite) Low Resolution Spectrograph and

Send offprint requests to: menes@graal.univ-montp2.fr

1 Table 2 is only available in electronic form at the CDS via anonymous ftp to cdsarc.u-strasbg.fr (130.79.128.5) or via http://cdsweb.u-strasbg.fr/Abstract.html

2 The term "long-period variables" is used here to cover Miras and semi-regular variables (according to the definition given in the GCVS, see below). The $\mathrm{OH} / \mathrm{IR}$ stars are excluded from this study.
Point Source Catalog. The period seems to be a particularly important characteristic of LPVs. Campbell (1925) first showed that a statistical relationship exists between the period and the shape of the light curve. The period is also correlated to the infrared excess due to circumstellar dust (De Gioia-Eastwood et al. 1981; Jura 1986), and to the expansion velocity (Ukita 1982; Sivagnanam et al. 1989). These relations could be connected to the link between the period and the estimated mass loss rate (Wood 1990). Whitelock et al. (1986, 1991) have shown that larger mass loss is correlated to longer period and larger amplitude. Furthermore, it has been mentioned in many works that LPVs with more asymmetric light curves lose mass more rapidly than those with symmetric ones (see the review by Jura \& Kleinmann 1992a).

As established previously by Feast (1963), and confirmed by many recent works (for example, Feast 1989; Kerschbaum \& Hron 1992; Jura \& Kleinmann 1992a, 1992b; Jura et al. 1993; Luri et al. 1996), LPVs exhibit distinctive kinematic properties and thus belong to different stellar populations. The period of the star could be a discriminant parameter.

These relationships are still not exactly understood, but it is clear that the period, amplitude, and asymmetry of the light curves of LPVs seem to be closely connected to their pulsation properties and therefore to their evolution. Hence these parameters might be used to define an automatic classification and to identify homogeneous groups of LPVs.

These evolved AGB-stars include three spectral types: $\mathrm{M}, \mathrm{S}$, and $\mathrm{C}$, depending on the abundance ratio $\mathrm{C} / \mathrm{O}$ in their surface layers and on their spectroscopic characteristics. S-stars are probably intermediate objects. A similar dichotomy also exists in their circumstellar material; the silicate and $\mathrm{SiC}$ dust features are observed respectively in the oxygen-rich (M-type and possibly S-type) and carbon-rich Miras. This can explain why IRAS colors alone are not sufficient to identify C LPVs. To distinguish 
carbon- from oxygen-rich stars, Epchtein et al. (1987) propose to use the $K$ - and $L$-band photometric fluxes.

The purpose of this paper is to study the mean light curve parameters of LPVs, to classify these stars according to these parameters, and to use this classification together with IRAS colors to discriminate between carbonand oxygen-rich LPVs (Sect. 6).

After a brief description of the data in Sects. 2 and 3, we present in Sect. 4 some statistical results on mean parameters of light curves obtained from the AAVSO visual data over 75 years for a sample of 355 LPVs. In Sect. 5 we present an automatic classification of our LPV sample based on the characteristics of their mean light curve, examine properties of stars belonging to each cluster and the link with previous classifications. Finally, we examine how the IRAS colors and our classification enable us to recognize carbon-rich LPVs.

\section{Basic data}

The AAVSO has compiled observed times and visual magnitudes for maxima and minima of about $400 \mathrm{LPVs}$ since 1900 (Mattei 1988). Our study is performed on these data which were also used in the preparation of the HIPPARCOS mission. Some stars were excluded from the sample either because of the peculiar shape of their light curves (e.g, double maxima: R Cen, R Nor, RZ Cyg, etc.), or due to insufficient data. The final sample contains 355 stars.

For each star the following parameters of the light curve were derived:

- Period, $P$ : time (in days) from one maximum to the following one.

- Amplitude, A: magnitude difference between the maximum and the minimum.

- Asymmetry factor, $f$ : the ratio of the increasing time (from minimum to the following maximum) to the period.

- Magnitude, Max, at maximum brightness.

- Magnitude, min, at minimum brightness.

- Their respective standard deviations: $\sigma_{\mathrm{P}}, \sigma_{\mathrm{A}}, \sigma_{\mathrm{f}}, \sigma_{\mathrm{M}}$, $\sigma_{\mathrm{m}}$.

\section{Additional data}

For each star we have collected the following additional information. Table 1 gives the distribution of the stars according to these additional data.

\subsection{Variability type}

The General Catalog of Variable Stars (Kholopov et al. 1985, here after GCVS) divides long period red variables into two groups, those with amplitudes greater or less than 2.5 mag. The small-amplitude stars are often called "SemiRegular" and the larger amplitude ones "Miras". It ap- pears that this amplitude rule is not followed very strictly and that the division between these two groups of LPVs is somewhat artificial (see also Kerschbaum \& Hron 1992). However, strong correlation between amplitude and emission features at some specific phase in the visible spectra has been found earlier. Using emission line information, Feast (1972) obtained a similar but not exactly identical division.

\subsection{Spectral type}

Both Mira and SR variables include M-, S-, and C-type stars. The $\mathrm{C} / \mathrm{O}$ abundance ratio was the first criterion discriminating the $\mathrm{M}$ type (also called oxygen-rich stars, $\mathrm{C} / \mathrm{O} \lesssim 1)$ and the $\mathrm{S}$ stars $(\mathrm{C} / \mathrm{O} \sim 1)$, from the $\mathrm{C}$ type $(\mathrm{C} / \mathrm{O} \gtrsim 1$, also called carbon stars). This criterion corresponds to the chemical composition of the surface layers. The second criterion was based on their spectral characteristics (for a review, see Jaschek \& Jaschek 1987). Let us remark that the classification of the $\mathrm{C}$ stars is less certain than that of $\mathrm{M}$ stars, while $\mathrm{S}$ stars are the most difficult to classify.

Intermediate objects also exist, as those between $\mathrm{S}$ and M stars, called MS and SM stars, or those between C and S stars, called SC and CS. Less common are those between C and M stars, called CM and MC stars (for AGB chemical composition, see Lambert 1988).

\subsection{Maser emissions}

Masers from late type stars are essentially emitted by three molecules (Elitzur 1980): OH (hydroxyl radical), $\mathrm{SiO}$ (silicon monoxide), and $\mathrm{H}_{2} \mathrm{O}$ (water). Detection of such maser emission depends both on its intensity and on the distance of the star (Dickinson et al. 1978), thus nondetection of emission can be due either to non-emission by the star or to greater distance of an emitter. Also, the possibility that some stars have asymmetric shells (perhaps because of an asymmetric mass loss) can make detection of maser emission dependent on the star's orientation. Furthermore, in a binary system the companion could supress the maser emissions (Lewis et al. 1987). Despite all this, maser emissions are statistically meaningful.

The pumping mechanism responsible for $\mathrm{OH}$ masers is associated with the IR radiation. The properties of type I OH LPVs (characterized by main line emissions) and type II (showing strong satellite emission lines) have been studied earlier and some correlations have been found (Bower \& Kerr 1977; Ukita 1982; Sivagnanam et al. 1989), including a strong correlation with mass loss. So, there is some evidence for the following sequence of increasing mass loss rates: (non $\mathrm{OH})-($ type $\mathrm{I} \mathrm{OH})-($ type II OH)$(\mathrm{OH} / \mathrm{IR})$ stars.

$\mathrm{SiO}$ maser emissions seem to be an atmospheric phenomenon (coming probably from the inner regions of the envelope) and thus provide direct information on the 
motion in it (Elitzur 1981; McIntosh et al. 1989). Hall et al. (1990) detected changes in the intensity of the $\mathrm{SiO}$ masers. Some suggest that the maser luminosity may depend strongly on the mass loss rate (cf. Bowers 1985).

The $\mathrm{H}_{2} \mathrm{O}$ maser emissions are still the less-studied ones; the difficulties come from the strong variability of their profiles (e.g. Bowers et al. 1993). Even so, a relationship has been suggested between these variations and the mass loss rate (Engels \& Lewis 1990; Bowers \& Johnston 1994).

\section{Parameters of the light curves}

\subsection{Mean parameters}

Mean parameters have been calculated for the light curve of each star of our sample using 75 years of AAVSO data. Their average values and standard deviations are given in Table 2. The information in the columns is as follows:

- Column (1): The name of the star.

- Columns (2) to (5): The available number of entries for the periods, maxima, minima, and visual amplitudes, respectively.

- Column (6): The mean period.

- Column (7): The mean asymmetry factor.

- Column (8): The mean magnitude at maximum brightness.

- Column (9): The mean magnitude at minimum brightness. We note that the average magnitude at minimum for some stars may have low reliability due to the observationnal limit that can be reached at this phase. The minima of these stars should be treated with caution; the standard deviations can give a confidence level.

- Column (10): The mean visual amplitude. On average, the estimation of the amplitude from either maximum to the following minimum or else from minimum to the following maximum, lead to the same value. Note, however, that low reliability of the minima causes a low confidence of the corresponding amplitude value. Hence, some mean amplitudes should be treated with caution.

- Columns (11) to (15): The standard deviations of, respectively, period, asymmetry factor, magnitudes at maximum and minimum, and amplitude.

- Columns (16) to (17). Variability type and spectral type taken from the GCVS.

\subsection{Properties of some standard deviations}

Figure 1 shows that, except the semiregular variables of M spectral type, the other LPVs roughly obey $\sigma_{\mathrm{P}} \sim$ constant for $P \lesssim 275$ days and a relation $\sigma_{\mathrm{P}} / P \sim$ constant for longer periods. This could be related to the fact that longer-period stars generally exhibit more complex pulsation, inducing more important mass-loss.

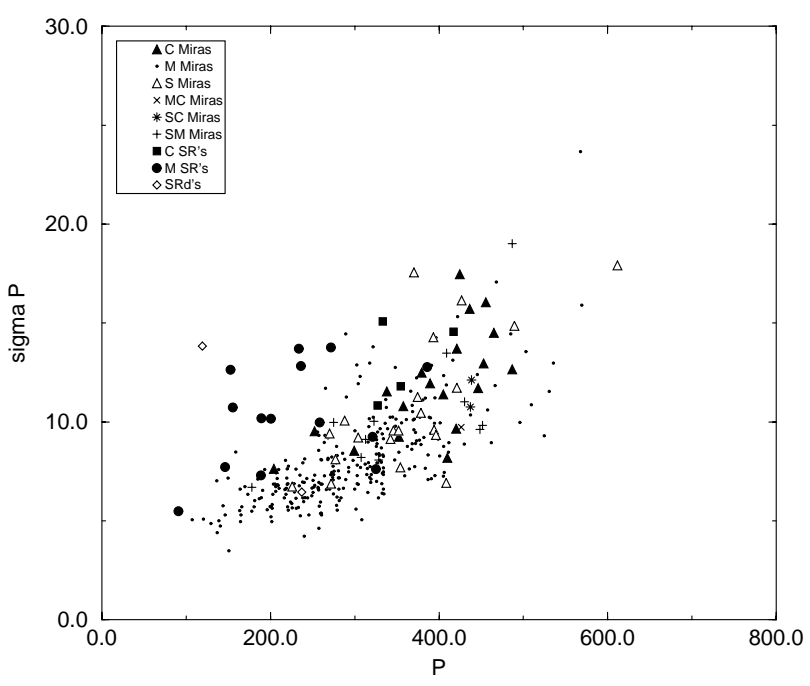

Fig. 1. The standard deviation of the period as a function of the period length for the 355 LPVs. Dots are for M Miras, triangles for S Miras, filled triangles for C Miras, cross for MC Miras, asterisks for CS or SC Miras, plus for SM or MS Miras, filled circles for M SRa's or SRb's, filled squares for C SR's, diamonds for SRd's

\subsection{Period-amplitude-asymmetry relations}

Reviews about the importance of these parameters are given by Whitelock et al. (1991) and by Jura \& Kleinmann (1992a). We have summarized our results in Fig. 2. We distinguish between Miras and SRs, and the various spectral types.

As is well-known, we find in general that carbon-rich Miras have large periods with small amplitudes, and that only a third of them show significant asymmetry in their light curves. S-Miras have large periods and amplitudes. Only S-Miras show a clear tendency of increasing amplitude with period. The "continuity" between SR's and Cstars is to be noted.

\section{Automatic classification}

We have attempted to classify the 355 LPVs based on three parameters characterizing their mean light curves: period $P$, amplitude $A$, and asymmetry factor $f$. Mennessier (1985) realized such classification on a smaller sample covering a shorter time span of observations. Ludendorff (1928) made an earlier classification of LPVs based on the shape of their visual light curves (see also Mattei 1983).

We used a dynamical clustering, which is a nonhierarchical analysis (Murtagh \& Heck 1987), and point out that the optimum number of clusters from the 
Table 1. Summary statistics

\begin{tabular}{|c|c|c|c|c|c|c|c|c|c|c|c|c|c|c|c|}
\hline & & \multicolumn{2}{|c|}{$\begin{array}{c}\text { Variable } \\
\text { type }\end{array}$} & \multicolumn{3}{|c|}{$\begin{array}{l}\text { Spectral } \\
\text { type }\end{array}$} & \multicolumn{3}{|c|}{$\begin{array}{c}\mathrm{OH} \\
\text { emission }\end{array}$} & \multicolumn{3}{|c|}{$\begin{array}{c}\mathrm{SiO} \\
\text { emission }\end{array}$} & \multicolumn{3}{|c|}{$\begin{array}{c}\mathrm{H}_{2} \mathrm{O} \\
\text { emission }\end{array}$} \\
\hline & & Mira & SR & $\mathrm{M}$ & $\mathrm{S}$ & $\mathrm{C}$ & $\mathrm{Y}$ & $\mathrm{N}$ & $?$ & $\mathrm{Y}$ & $\mathrm{N}$ & $?$ & $\mathrm{Y}$ & $\mathrm{N}$ & $?$ \\
\hline $\begin{array}{c}\text { Variable } \\
\text { type }\end{array}$ & $\begin{array}{c}\text { Mira } \\
\text { SR }\end{array}$ & $\begin{array}{r}335 \\
-\end{array}$ & 20 & & & & & & & & & & & & \\
\hline $\begin{array}{l}\text { Spectral } \\
\text { type }\end{array}$ & $\begin{array}{c}\mathrm{M} \\
\mathrm{S} \\
\mathrm{C}\end{array}$ & $\begin{array}{r}286 \\
27 \\
19\end{array}$ & $\begin{array}{r}14 \\
0 \\
4\end{array}$ & $\begin{array}{r}300 \\
- \\
-\end{array}$ & $\begin{array}{r}27 \\
-\end{array}$ & 23 & & & & & & & & & \\
\hline $\begin{array}{c}\mathrm{OH} \\
\text { maser } \\
\text { emission }\end{array}$ & $\begin{array}{l}\mathrm{Y} \\
\mathrm{N} \\
?\end{array}$ & $\begin{array}{r}52 \\
171 \\
111\end{array}$ & $\begin{array}{r}0 \\
6 \\
14\end{array}$ & $\begin{array}{r}52 \\
172 \\
76\end{array}$ & $\begin{array}{r}0 \\
2 \\
25\end{array}$ & $\begin{array}{r}0 \\
2 \\
20\end{array}$ & $\begin{array}{r}52 \\
- \\
-\end{array}$ & $\begin{array}{r}177 \\
-\end{array}$ & 125 & & & & & & \\
\hline $\begin{array}{c}\mathrm{SiO} \\
\text { maser } \\
\text { emission }\end{array}$ & $\begin{array}{l}\mathrm{Y} \\
\mathrm{N} \\
?\end{array}$ & $\begin{array}{r}94 \\
108 \\
133\end{array}$ & $\begin{array}{r}1 \\
5 \\
14\end{array}$ & $\begin{array}{r}89 \\
107 \\
104\end{array}$ & $\begin{array}{r}5 \\
5 \\
17\end{array}$ & $\begin{array}{r}1 \\
1 \\
21\end{array}$ & $\begin{array}{r}44 \\
7 \\
2\end{array}$ & $\begin{array}{l}41 \\
98 \\
38\end{array}$ & $\begin{array}{r}10 \\
8 \\
107\end{array}$ & $\begin{array}{r}95 \\
- \\
-\end{array}$ & $\begin{array}{r}113 \\
-\end{array}$ & 147 & & & \\
\hline $\begin{array}{c}\mathrm{H}_{2} \mathrm{O} \\
\text { maser } \\
\text { emission }\end{array}$ & $\begin{array}{l}\mathrm{Y} \\
\mathrm{N} \\
?\end{array}$ & $\begin{array}{r}69 \\
168 \\
98\end{array}$ & $\begin{array}{l}2 \\
9 \\
9\end{array}$ & $\begin{array}{r}71 \\
147 \\
82\end{array}$ & $\begin{array}{r}0 \\
20 \\
7\end{array}$ & $\begin{array}{r}0 \\
9 \\
14\end{array}$ & $\begin{array}{r}36 \\
13 \\
4\end{array}$ & $\begin{array}{r}32 \\
109 \\
36\end{array}$ & $\begin{array}{r}3 \\
55 \\
67\end{array}$ & $\begin{array}{r}51 \\
41 \\
3\end{array}$ & $\begin{array}{l}15 \\
69 \\
29\end{array}$ & $\begin{array}{r}5 \\
67 \\
75\end{array}$ & $\begin{array}{r}71 \\
- \\
-\end{array}$ & 177 & 107 \\
\hline
\end{tabular}

inertia criterion seems to be six. From several random initial conditions the best partition was extracted.

Each cluster of the best partition is characterized by its position in $(P, A, f)$-space as given in Table 3 . It turns out that $P$ and $A$ are independent parameters in this classification, but that $f$ appears in linear combinations with $P$ and $A$. The classification is realized in terms of the four variables $P, A, 100 f-5.9 A$, and $100 f-0.0022 P$, which are discriminant parameters, and are easily determined from observations.

\subsection{Properties of stars in each cluster}

\subsubsection{Spectral types}

The number of stars of each cluster according to their spectral type is given in Table 4 . It is notable that $80 \%$ of $\mathrm{C}$ stars belong to cluster C3. Still, this cluster also contains $\mathrm{M}$ stars, so this classification is not sufficient to discriminate C-rich from O-rich LPVs. Note the similarity between clusters $\mathrm{C} 2$ and $\mathrm{C} 4$ (in which there are no carbon stars), as well as that between C3 and C5 (which contain most of the $\mathrm{C}$ stars and SRs respectively).

\subsubsection{Maser emission}

The contingency table of maser emissions and our clustering is given in Table 5. In each column the number of stars is indicated, followed (in parentheses) by 100 times the ratio of the observed frequency to that which is expected if the variables are independent:

$p_{i j}=100 n_{i j} / f_{i j}=100\left(n_{i j} / n_{i .} n_{. j}\right) n$,

where $n$ is the total number of individuals, $n_{i}$. the total number in line $i$ and $n_{\text {.j }}$ the total in column $j$. Significantly high and low ratios are indicated by one or two underlines respectively.

As can be predicted, maser emission has not been searched for among $\mathrm{C}$ stars. The significant frequencies confirm that:

1. $\mathrm{OH}$ emission is related to longer period and asymmetric light-curves ( $\mathrm{C} 1$ and $\mathrm{C} 6$ ) and not to short-period variables (C2 and $\mathrm{C} 5)$.

2. $\mathrm{SiO}$ emission is related to longer period and large amplitude (C6 and $\mathrm{C} 4$ ) and not with short periods (C2 and $\mathrm{C} 5)$. 
Table 3. The characteristics of the six best clusters as achieved from an automatic classification (dynamical clustering), $n$ is the number of stars belonging to each cluster

\begin{tabular}{|c|c|cccc|}
\hline $\begin{array}{c}\text { Best } \\
\text { cluster }\end{array}$ & $n$ & $P$ & $A$ & $100 \mathrm{f}-5.9 A$ & $100 f-0.022 P$ \\
\hline & & & & & \\
C2 & 110 & $<\sim 275$ & $>3.7$ & $>10.5 \&<26$ & - \\
$\mathrm{C} 4$ & 67 & $>\sim 275$ & $>3.7$ & $>10.5 \&<26$ & $>35$ \\
$\mathrm{C} 1$ & 54 & $>\sim 275$ & $<4.7$ & $>10.5 \&<26$ & $<35$ \\
$\mathrm{C} 6$ & 51 & $>\sim 275$ & $>4.7$ & $<10.5$ & $<35$ \\
$\mathrm{C} 5$ & 37 & $<\sim 275$ & $<3.7$ & $>26$ & - \\
$\mathrm{C} 3$ & 35 & $>\sim 275$ & $<3.7$ & $>26$ & $>35$ \\
& & & & & \\
\hline
\end{tabular}

Table 4. Number of stars in each cluster according to different types

\begin{tabular}{|c|cccccc|}
\hline Type & C1 & C2 & C3 & C4 & C5 & C6 \\
\hline O rich & & & & & & \\
Miras & 48 & 106 & 12 & 59 & 25 & 40 \\
SRs & 1 & & 3 & & 12 & \\
C rich & & & & & & \\
Miras & 4 & & 15 & & 1 & 1 \\
SRs & & & 4 & & & \\
S & 1 & 4 & 1 & 8 & & 10 \\
\hline
\end{tabular}

Table 5. Contingency table of our classification and maser emissions. $Y$ and $N$ respectively signify a "yes" or "no" to maser detection

\begin{tabular}{|c|c|c|c|c|c|c|c|c|c|c|c|c|c|}
\hline & \multicolumn{6}{|c|}{$\mathrm{OH}$ emission } & \multicolumn{6}{|c|}{ SiO emission } & \multirow{2}{*}{$\begin{array}{r}\text { Tot. by } \\
\text { line }\end{array}$} \\
\hline & & $Y$ & & V & & $?$ & & $Y$ & & $\mathrm{~N}$ & & ? & \\
\hline C3 & 3 & (57) & & $(69)$ & 20 & $(\underline{162})$ & 7 & $(75)$ & 3 & $(\underline{\underline{27}})$ & 25 & $(\underline{172})$ & 35 \\
\hline C5 & 1 & $(\underline{\underline{17}})$ & 25 & $(\underline{132})$ & 12 & $(90)$ & 0 & $(\underline{0})$ & 15 & $(\overline{24})$ & 23 & $(\underline{146})$ & 38 \\
\hline $\mathrm{C} 4$ & 10 & $(\overline{100})$ & 38 & (114) & 19 & $(81)$ & 26 & $(\underline{14 \overline{5}})$ & 17 & $(80)$ & 24 & $(87)$ & 67 \\
\hline $\mathrm{C} 2$ & 3 & $(\underline{18})$ & 76 & $(\underline{139})$ & 31 & $(80)$ & 15 & $(\underline{51})$ & 58 & $(\underline{166})$ & 37 & $(81)$ & 110 \\
\hline $\mathrm{C} 1$ & 14 & $(\underline{174})$ & 14 & $(\underline{\underline{52}})$ & 26 & $(\underline{137})$ & 14 & $(\overline{97})$ & 13 & $(76)$ & 27 & (12) 1 & 54 \\
\hline C6 & 22 & $(\underline{289})$ & 12 & $(\underline{\overline{47}})$ & 17 & $(95)$ & 33 & $(\underline{242})$ & 7 & $(\underline{\underline{43}})$ & 11 & $(\underline{\underline{52}})$ & 51 \\
\hline $\begin{array}{l}\text { Tot. by } \\
\text { column }\end{array}$ & 53 & & 177 & & 125 & & 95 & & 113 & & 147 & & $\begin{array}{r}\text { Toт. } \\
355\end{array}$ \\
\hline
\end{tabular}



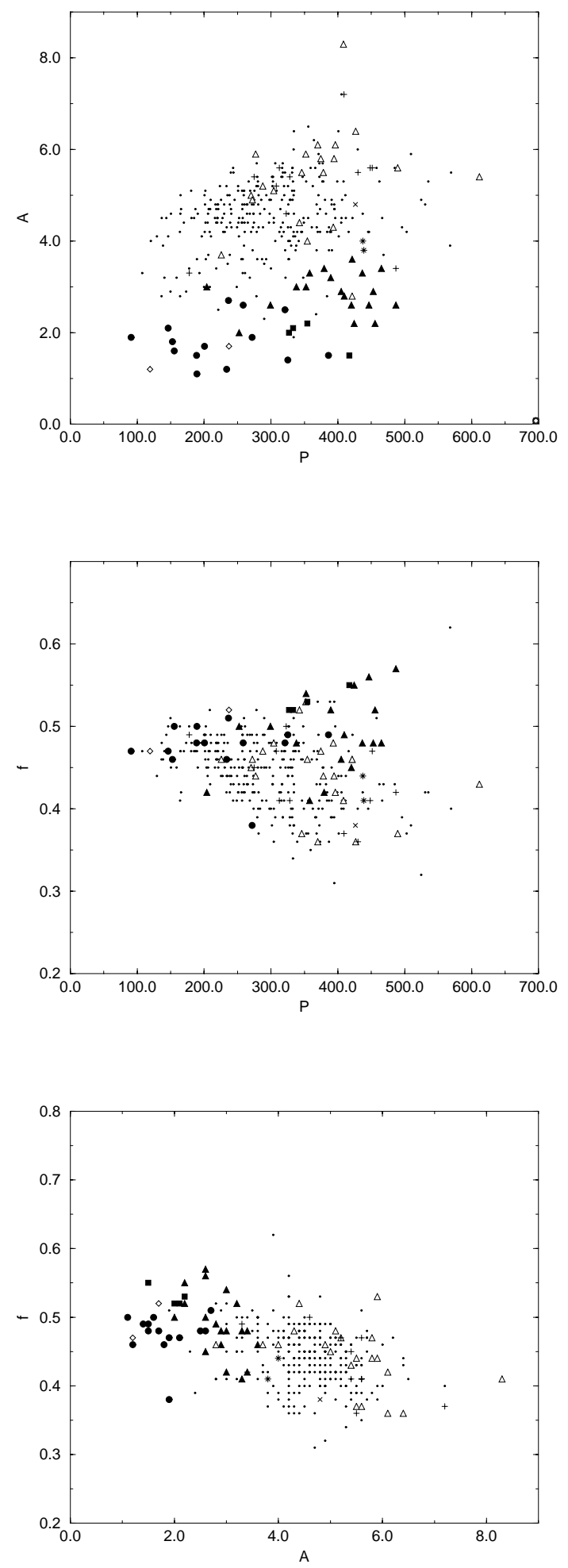

Fig. 2. Amplitude-period-asymmetry relations for the 355 LPVs discriminating by variability and spectral types. The symbols are the same as in Fig. 1

\subsubsection{Other classifications}

Automatic classifications using the dispersions $\sigma_{\mathrm{M}}, \sigma_{\mathrm{m}}$, $\sigma_{\mathrm{A}}$ were performed by Brito et al. (1992). The aim of this paper was to check a method of symbolic clustering by comparing it to a classical method. Their results led to a six-cluster classification; the contingency table with ours is given in Table 6 (the numbers have the same meaning as in Table 5). We conclude that:

1. S2 and S6, whose members are characterized by small or mean values of the three dispersions, are related to C3 and C5. This agrees with known properties of SR and $\mathrm{C}$ stars.

2. Mean or high values of dispersions (S4, S1, S5, S3) are mainly associated with clusters $\mathrm{C} 1$ and $\mathrm{C} 6$, which contain the most asymmetric light-curves.

\section{IRAS colors and light-curve clustering}

As we have emphasized, the difference between carbonand oxygen-rich stars depends on several parameters (the abundance of specific elements, the evolutionary state of the star, its chemical composition, its mass, etc.) to which the pulsation and the light curve are related. We have just seen that the light curve parameters are not sufficient to distinguish $\mathrm{C}$ stars. These parameters are mainly linked to the stellar rather than circumstellar properties. The properties of their circumstellar envelopes differ, but it is well known that parameters linked principally to the circumstellar medium, such as IRAS colors, also are not sufficient to distinguish $\mathrm{C}$ stars. Yet it may be that the combination of both sets of parameters, respectively reflecting the stellar and circumstellar properties, could be used to distinguish carbon- from oxygen-rich LPVs. Therefore we now look jointly at light curve parameters and IRAS colors.

\subsection{Our sample}

Figure 3 shows the classical ([12] - [25], [25] - [60]) IRAS color-color diagram separately for each cluster found by our light-curve classification. Only IRAS fluxes with a good quality (3) are considered. It can be seen that:

1. non-C miras belonging to $\mathrm{C} 3$ have IRAS colors different from the $\mathrm{C}$ miras belonging to $\mathrm{C} 3$,

2. non-C miras with IRAS colors in the range of the $\mathrm{C}$ miras belong to clusters other than C3.

This indicates that by considering both IRAS colors and light-curve classification, we can discriminate C-rich from O-rich LPVs.

\subsection{GCVS sample}

We have now to check if this result is robust or only due to a peculiarity of our sample. So we selected all miras in the GCVS with IRAS colors of quality 3 . To generate this 

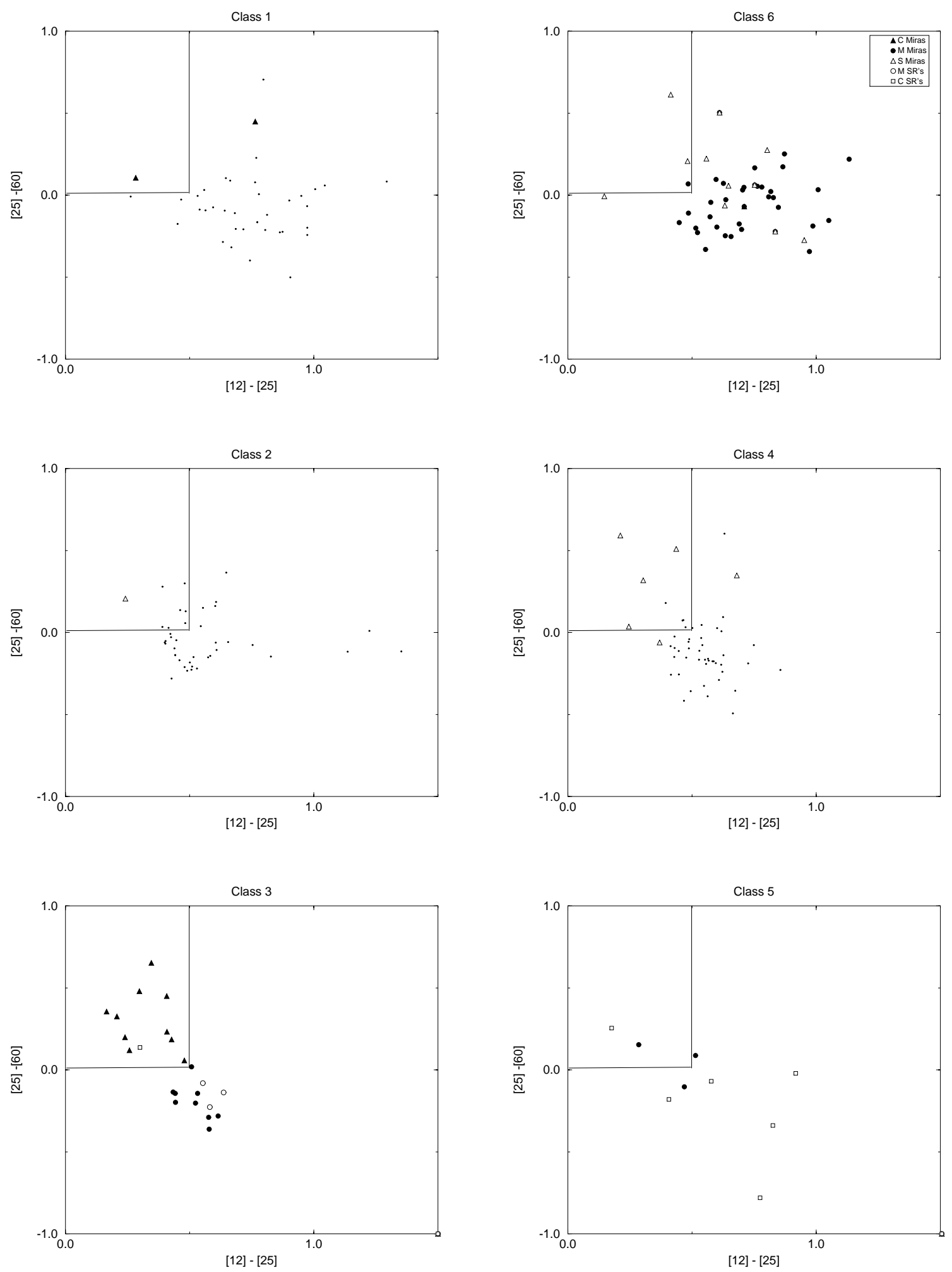

Fig. 3. IRAS color-color diagram for each cluster 
Table 6. Contingency table of our classification and the one obtained from the dispersions of light curve parameters

\begin{tabular}{|c|c|c|c|c|c|c|c|c|c|c|c|c|c|}
\hline & \multicolumn{2}{|c|}{$\mathrm{S} 4$} & \multicolumn{2}{|c|}{$\mathrm{S} 1$} & \multicolumn{2}{|c|}{$\mathrm{S} 2$} & \multicolumn{2}{|c|}{ S6 } & \multicolumn{2}{|c|}{ S5 } & \multicolumn{2}{|c|}{ S3 } & $\begin{array}{r}\text { Tot. by } \\
\text { line } \\
\end{array}$ \\
\hline C3 & 4 & (59) & 4 & (52) & 15 & $(\underline{188})$ & 7 & (91) & 3 & (132) & 2 & (78) & 35 \\
\hline C5 & 1 & $(\underline{\underline{13}})$ & 7 & (84) & 12 & (138) & 17 & (204) & 0 & $(\underline{\underline{0}})$ & 1 & $(\underline{\underline{36}})$ & 38 \\
\hline $\mathrm{C} 4$ & 15 & $(\overline{15})$ & 11 & (75) & 21 & (137) & 12 & $(81)$ & 2 & $(4 \overline{\overline{6}})$ & 6 & $(\overline{122})$ & 67 \\
\hline $\mathrm{C} 2$ & 18 & $(84)$ & 29 & (120) & 27 & (108) & 32 & (132) & 0 & $(\underline{\underline{0}})$ & 4 & $(50)$ & 110 \\
\hline $\mathrm{C} 1$ & 9 & $(86)$ & 19 & $(\underline{160})$ & 2 & $(\underline{\underline{16}})$ & 7 & $(59)$ & 10 & $(\underline{28 \overline{6}})$ & 7 & $(\underline{177})$ & 54 \\
\hline C6 & 22 & $(\underline{222})$ & 8 & $(71)$ & & $(\underline{\underline{\underline{34}}})$ & 3 & $(\underline{\underline{27}})$ & 8 & $(\underline{242})$ & 6 & (161) & 51 \\
\hline $\begin{array}{l}\text { Tot. by } \\
\text { column }\end{array}$ & 69 & & 78 & & 81 & & 78 & & 23 & & 26 & & $\begin{array}{r}\text { Toт. } \\
355\end{array}$ \\
\hline
\end{tabular}
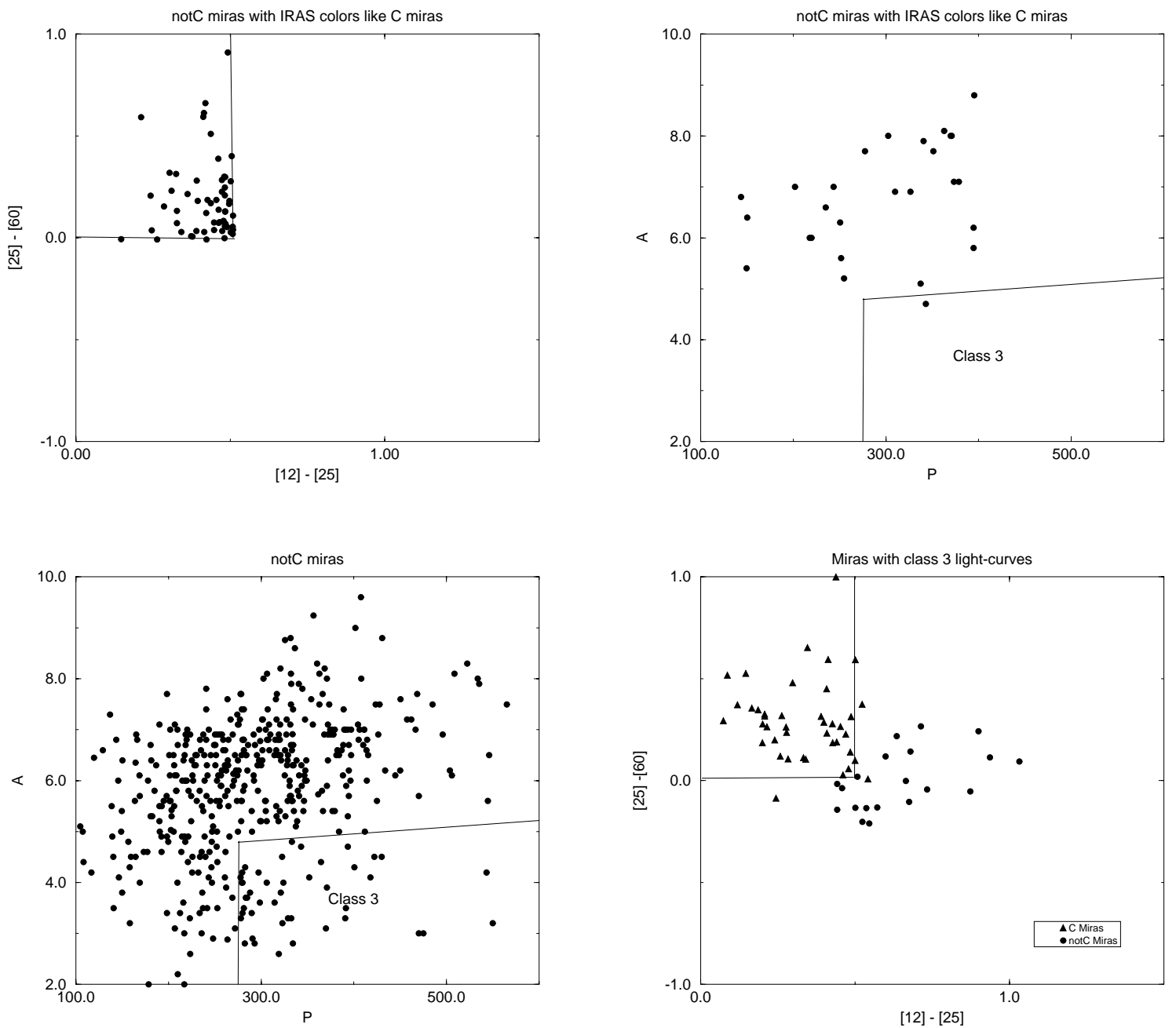

Fig. 4. IRAS color-color diagram and location on $(P, A)$ plane for different samples 
sample we used the database ASTRID (Mennessier et al. 1996).

The results are displayed in Fig. 4. It must be noted that to compute the amplitude, we must compensate for the fact that the GCVS indicates not the mean maxima and minima, but the most luminous and the least luminous observed magnitudes. To define the corresponding limits of C3, we must apply statistical corrections (Mennessier \& Guibert 1983) to the GCVS data:

$\max _{\text {mean }}=\max _{\mathrm{GCVS}}+0.32+0.11(P / 100)$,

and

$\min _{\text {mean }}=\min _{\mathrm{GCVS}}-0.6$.

We confirm that:

1. miras of $\mathrm{S}$ and $\mathrm{M}$ spectral types with IRAS colors like the ones of carbon miras do not belong to C3

2. non-C miras belonging to $\mathrm{C} 3$ have IRAS colors different from $\mathrm{C}$ miras belonging to this cluster.

Hence we verify our previous conclusion: combining IRAS colors with our classification enables us to distinguish carbon-rich from oxygen-rich miras.

\section{Discussion and outlook}

This paper is based on a statistical study of observations over an exceptionaly long period of time. We confirm previously known results that can help explain many other observational data and constrain the models.

Our main result is to produce a classification of LPVs from relatively easily observable parameters of their light curves that can be used jointly with IRAS colors to distinguish carbon- from oxygen-rich stars. One of its strengths is that all parts of these stars (stellar and circumstellar media, extended envelope, and their couplings) are taken into account.

Acknowledgements. We express our thanks and appreciation to variable star observers around the world whose observations over decades have make this work possible. J.A. Mattei acknowledges gratefully NASA grant NA6W-1493, which partially supported this work. We thank C. Mercier for his help in making the figures, the staff of INRIA for authorizing use of SICLA to do the classification, and G. Foster for assistance in preparing the manuscript.

\section{References}

Bowers P.F., 1985, in: Mass Loss From Red Giants, Morris M. and Zuckerman B. (eds.). Dordrecht, Reidel, p. 189

Bowers P.F., Kerr F.J., 1977, A\&A 57, 115

Bowers P.F., Claussen M.J., Johnston K.J., 1993, AJ 105, 284

Bowers P.F., Johnston K.J., 1994, ApJS 92, 189

Brito P., Mennessier M.O., Boughaleb H., 1992, in: Xth compstat
Campbell L., 1955, Studies of Long Period Variables, AAVSO ed.

DeGioia-Eastwood K., Hackwell J.A., Grasdalen G.L., Gehrz R.D., 1981, ApJ 245, L75

Dickinson D.F., Synder L.E., Brown L.W., Buhl D., 1978, AJ 83,36

Elitzur M., 1980, in: Physical Processes in Red Giants, Iben I. and Renzini A. (eds.). Dordrecht, Reidel, p. 363

Elitzur M., 1981, ApJ 240, 553

Engels D., Lewis B.M., 1990, Proc.: From Miras to Planetary Nebulae, Mennessier M.O. and Omont A. (eds.). Editions Frontières, Gif sur Yvette, p. 250

Epchtein N., Le Bertre T., Lépine J., et al., 1987, A\&AS 71, 39

Feast M.W., 1963, MNRAS 125, 367

Feast M.W., 1972, QJRAS 13, 191

Feast M.W., 1989, in: The Use of Pulsating Stars in Fundamental Problems of Astronomy, Schmidt (ed). Cambridge University Press

Habing H.J., 1990, Proc.: From Miras to Planetary Nebulae, Mennessier M.O. and Omont A. (eds). Editions Frontières, Gif sur Yvette, p. 16

Habing H.J., 1996 A\&AR 7, 97

Hall P.J., Allen D.A., Troup E.R., Wark R.M., Wright A.E., 1990, MNRAS 243, 480

Jascheck M., Jascheck C., 1987, in: The classification of Stars, Jascheck M. and Jascheck C. (eds.). Cambridge Univ. Press Jura M., 1986, ApJ 303, 327

Jura M., Kleinmann S.G., 1992a, ApJS 79, 105

Jura M., Kleinmann S.G., 1992b, ApJS 83, 329

Jura M., Yamamoto A, Kleinmann S.G., 1993, ApJ 413, 298

Kerschbaum F., Hron J., 1992, A\&A 263, 97

Kholopov P.N., et al., 1985, General Catalogue of Variable Stars, 4th ed. Moscow: Moscow Pub. House

Lambert D.L., 1988, in: Evolution of Peculiar Red Giant Stars, Johnson H.R. and Zuckerman B. (eds.). Cambridge, p. 101

Lewis B.M., Eder J., Terzian Y., 1987, AJ 94, 1025

Ludendorff H., 1928, Handbuch der Astrophys. 6, 49

Luri X., Mennessier M.O., Figueras F., Torra J., 1996, A\&A (in press)

McIntosh G.C., Predmore C.R., Moran J.M., et al., 1989, ApJ 337,934

Mattei J.A., 1983, AAVSO Report 38, ed. AAVSO. Cambridge

Mattei J.A., 1988, in: Scientific aspects of the Input Catalogue Preparation, Torra J. and Turon C. (eds.) p. 379

Mennessier M.O., 1985, A\&A 144, 463

Mennessier M.O., Guibert J., 1983, A\&A 128, 69

Mennessier M.O., Mennessier G., Tack J., 1996, ASTRID database http://www.graal.univ-montp2.fr

Murtagh F., Heck A., 1987, Multivariate Data Analysis. Kluwer

Sivagnanam P., Le Squeren A.M., Foy F., 1989, A\&A 211, 341

Ukita N., 1982, A\&A 112, 167

Whitelock P.A., Pottasch S.R., Feast M.W., 1986, in: Late Stages of Stellar Evolution, Kwok S. and Pottasch S.R. (eds.). Dordrecht, Reidel, p. 269

Whitelock P.A., Feast M., Catchpole R., 1991, MNRAS 248, 276

Wood P.R., 1990, Proc.: From Miras to Planetary Nebulae, Mennessier M.O. and Omont A. (eds.). Editions Frontières, Gif sur Yvette, p. 67 